\title{
A Liouville type theorem for a class of anisotropic equations
}

\author{
L. Barbu and C. Enache
}

\begin{abstract}
In this paper we are dealing with entire solutions of a general class of anisotropic equations. Under some appropriate conditions on the data, we show that the corresponding equations cannot have non-trivial positive solutions bounded from above.
\end{abstract}

\section{Introduction}

The classical Liouville Theorem states that any harmonic function on the whole Euclidian space $\mathbb{R}^{N}, N \geq 2$, which is bounded from one side, must be identically constant. Nowadays, it is well known that this property is not a prerogative of harmonic functions, since it is also shared by bounded (from below and/or above) entire solutions to more general elliptic partial differential equations (we refer the reader to the survey paper of A. Farina [7]). Some of these Liouville type results are obtained by using the maximum principle, which is the best tool employed to get a priori pointwise estimates on the gradient of the solutions (see, for instance, the seminal papers of L.A. Peletier and J. Serrin [13], B. Gidas and J. Spruck [10], L. Modica [12] and the extensions known to these results). In this paper, adapting an idea from the works of L. Modica [12] and L.A. Caffarelli, N. Garofalo and F. Segala [1], we are going to employ again the maximum principle to establish a new Liouville type theorem for entire solutions of a general class of anisotropic equations.

Key Words: Liouville theorem, anisotropic equations, maximum principles, entire solutions. 2010 Mathematics Subject Classification: Primary 35J25; Secondary 35B50.

Received: 27.07.2015

Accepted: 22.01.2016 
Let $p>1$ be a real constant and $F: \mathbb{R}^{N} \rightarrow[0, \infty), N \geq 2$, be a homogeneous function of degree 1 , with the following properties:

$$
\begin{aligned}
& F \in C_{\text {loc }}^{3, \alpha}\left(\mathbb{R}^{N} \backslash\{\mathbf{0}\}\right), \text { with } \alpha \in(0,1), \\
& F(\xi)>0 \text { for all } \xi \in \mathbb{R}^{N} \backslash\{\mathbf{0}\}, \\
& \text { Hess }\left(F^{p}\right) \text { is positive definite in } \mathbb{R}^{N} \backslash\{\mathbf{0}\} .
\end{aligned}
$$

Obviously, we also have $F(\mathbf{0})=0$, since $F$ is homogeneous and defined at the origin. Let us introduce the anisotropic p-Laplace operator, defined as follows:

$$
Q u:=\sum_{i=1}^{N} \frac{\partial}{\partial x_{i}}\left[F^{p-1}(\nabla u) F_{\xi_{i}}(\nabla u)\right] .
$$

We immediately remark the followings: when $F(\xi)=|\xi|, Q$ is the classical $p$ Laplace operator (see P. Lindqvist [11]), while when $p=2, Q$ is the anisotropic operator, also known as the Finsler-Laplace operator (see V. Ferone and B. Kawohl [9]). In this paper we investigate the following class of anisotropic equations:

$$
Q u+f(u)=0 \text { in } \mathbb{R}^{N},
$$

where the nonlinearity $f$ is a real differentiable function which satisfies

$$
f^{\prime}(t) \leq \begin{cases}(p-1) \frac{N+1}{N-p} \frac{f(t)}{t} \text { when } 1<p<N, & \\ L \frac{f(t)}{t} \text { when } p \geq N, & \text { for any } t>0,\end{cases}
$$

while $L$ is a nonnegative real constant.

The main result of this paper states:

Theorem 1.1. Assume that $u(\mathbf{x}) \in L^{\infty}\left(\mathbb{R}^{N}\right) \bigcap W_{\text {loc }}^{1, p}\left(\mathbb{R}^{N}\right)$ is a weak solution of equation (1.3), such that $\inf _{\mathbb{R}^{N}} u(\mathbf{x})>0$. Then $u(\mathbf{x})$ must be identically constant. As a consequence, if $f(t)$ has no positive root, then (1.3) has no weak solution $u(\mathbf{x}) \in L^{\infty}\left(\mathbb{R}^{N}\right) \cap W_{l o c}^{1, p}\left(\mathbb{R}^{N}\right)$, with $\inf _{\mathbb{R}^{N}} u(\mathbf{x})>0$.

We note here that L. D'Ambrosio and E. Mitidieri have also investigated recently in [3], [4] and [5] some interesting links between Liouville type theorems and the existence of uniform bounds of solutions of some nonlinear elliptic pde's. The operator studied in our paper and their generalizations are not explicitly studied in their papers, but their approach may be employed to 
get a similar Liouville type result under the following condition, which looks different than (1.4):

$$
\limsup _{t \rightarrow 0^{+}} \frac{f(t)}{t^{\frac{N(p-1)}{N-p}}}>0(\text { possibly } \infty) \text {, when } N>p .
$$

However, since the solution is assumed to be bounded away from 0 , their results still may be employed to get a result similar to Theorem 1 (see Section 4 , for more details).

The main ingredients of the proof are a maximum principle for an appropriate functional combination of $u(\mathbf{x})$ and $\nabla u(\mathbf{x})$, i.e. a kind of $P$-function in the sense of L.E. Payne (see the book of R. Sperb [15]), the translation invariance of equation (1.3) and some well-known $C^{1, \alpha}$ a priori estimates.

The outline of the paper is as follows. In Section 2 we establish a strong maximum principle for a $P$-function of the form $F^{p}(\nabla u(\mathbf{x})) / u(\mathbf{x})^{\beta}$, with $\beta$ to be appropriately chosen, while in Section 3 this new maximum principle is employed to prove Theorem 1.

For convenience, notice that throughout this paper the comma is used to indicate differentiation and the summation from 1 to $N$ is understood on repeated indices. Moreover, we adopt the following notations:

$$
\begin{aligned}
& F:=F(\nabla u), \\
& F_{i}:=F_{\xi_{i}}=\frac{\partial F}{\partial \xi_{i}} \text { for } i \in\{1, \ldots, N\}, \\
& a_{i j}(\nabla u)(\mathbf{x}):=\frac{\partial^{2}}{\partial \xi_{i} \partial \xi_{j}}\left(\frac{1}{p} F^{p}(\nabla u)\right)(\mathbf{x}) \\
& =F^{p-1} F_{i j}+(p-1) F^{p-2} F_{i} F_{j}, \\
& a_{i j k}(\nabla u)(\mathbf{x}):=\frac{\partial^{3}}{\partial \xi_{i} \partial \xi_{j} \partial \xi_{k}}\left(\frac{1}{p} F^{p}(\nabla u)\right)(\mathbf{x}) \text { for } i, j, k \in\{1, \ldots, N\} .
\end{aligned}
$$

\section{A maximum principle for an appropriate P-function}

In this section we are going to establish a strong maximum principle (see the book of M. H. Protter and H.F. Weinberger [14]) for the following appropriate P-function

$$
P(u ; \mathbf{x}):=\frac{F^{p}(\nabla u(\mathbf{x}))}{u^{\beta}(\mathbf{x})},
$$


where $u(\mathbf{x}) \in W_{l o c}^{1, p}\left(\mathbb{R}^{N}\right)$ is a weak solution to equation (1.3), while

$$
\beta:=\left\{\begin{array}{l}
p \frac{N-1}{N-p} \text { when } 1<p<N, \\
L \frac{p}{p-1} \frac{N-1}{N+1} \text { when } p \geq N .
\end{array}\right.
$$

We have:

Theorem 2.1. Suppose that the assumptions of Theorem 1.1 are satisfied. Let $\Omega \subset \mathbb{R}^{N}, N \geq 2$, be a bounded domain and assume in addition that $\inf _{\bar{\Omega}}|\nabla u(\mathbf{x})|>0$. If there exists $\mathbf{x}_{0} \in \Omega$ such that

$$
P\left(u ; \mathbf{x}_{0}\right)=\sup _{\mathbf{x} \in \Omega} P(u ; \mathbf{x})
$$

then $P(u ; \cdot)$ is identically constant in $\Omega$.

For the proof of Theorem 2.1, the following two lemmas will be very useful:

Lemma 2.2. If $F \in C^{3}\left(\mathbb{R}^{N} \backslash\{0\}\right)$ is a positive homegenous function of degree 1 , then we have

$$
\begin{aligned}
& F_{\xi_{i}}(\xi) \xi_{i}=F(\xi), \\
& F_{\xi_{i} \xi_{j}}(\xi) \xi_{i}=0, \quad \text { for any } \xi \in \mathbb{R}^{N} \backslash\{\mathbf{0}\} . \\
& F_{i j k}(\xi) \xi_{i}=-F_{j k}(\xi),
\end{aligned}
$$

For the proof of Lemma 2.2 we refer the reader to A. Farina and E. Valdinoci [8] (Lemma 3, Appendix).

Lemma 2.3. Assume that $u(\mathbf{x})$ is of class $C^{2}$ at the points where $\nabla u \neq \mathbf{0}$. Then, at such points, we have

$$
a_{i j} a_{k l} u_{i k} u_{j l} \geq \frac{\left(a_{i j} u_{i j}\right)^{2}}{N}+\frac{N}{N-1}\left[\frac{a_{i j} u_{i j}}{N}-(p-1) F^{p-2} F_{i} F_{j} u_{i j}\right]^{2} .
$$

For a proof of Lemma 2.3, in the case $p=2$, we refer the reader to G. Wang and C. Xia [16]. Using similar arguments one may easily prove inequality (2.5) for an arbitrary $p$.

We are now going to prove Theorem 2.1. The main idea of the proof is the construction of an elliptic second order differential inequality for the auxiliary 
function $P$, introduced in (2.1). The conclusion of the theorem will then follow immediately, as a direct consequence of Hopf's first maximum principle (see R. Sperbce [15]).

First of all, let us remark that, since $\operatorname{Hess}\left(F^{p}\right)$ is positive definite on $\mathbb{R}^{N} \backslash\{\mathbf{0}\}$, the anisotropic $p$-Laplace operator $Q$ is uniformly elliptic on $\mathbb{R}^{N} \backslash \mathrm{C}$, where $\mathcal{C}:=\left\{\mathbf{x} \in \mathbb{R}^{N} \mid \nabla u(\mathbf{x})=\mathbf{0}\right\}$. Moreover, since $F \in C_{\text {loc }}^{3, \alpha}\left(\mathbb{R}^{N} \backslash\{\mathbf{0}\}\right)$, Proposition 3.2 from M. Cozzi-A.Farina-E.Valdinoci [2] implies that a weak solution $u \in W_{\text {loc }}^{1, p}\left(\mathbb{R}^{N}\right)$ to equation $(1.3)$ is of class $C^{3}$ on $\mathbb{R}^{N} \backslash \mathcal{C}$. Therefore, the partial derivatives of $u(\mathbf{x})$, up to third order, are well defined on $\mathbb{R}^{N} \backslash \mathrm{C}$.

We now compute successively

$$
\begin{aligned}
& P_{i}=p F^{p-1} F_{k} u_{k i} u^{-\beta}-\beta F^{p} u_{i} u^{-\beta-1}, \\
& P_{i j}=p(p-1) F^{p-2} F_{l} F_{k} u_{k i} u_{l j} u^{-\beta}+p F^{p-1} F_{k l} u_{l j} u_{k i} u^{-\beta} \\
& +p F^{p-1} F_{k} u_{k i j} u^{-\beta}-\beta p F^{p-1} F_{k} u_{k i} u_{j} u^{-\beta-1} \\
& -\beta p F^{p-1} F_{k} u_{k j} u_{i} u^{-\beta-1}+\beta(\beta+1) F^{p} u_{i} u_{j} u^{-\beta-2} \\
& -\beta F^{p} u_{i j} u^{-\beta-1} \text {. }
\end{aligned}
$$

Next, making use of notations (1.5), we note that equation (1.3) may be rewritten as follows

$$
a_{i j} u_{i j}=\left[F^{p-1} F_{i j}+(p-1) F^{p-2} F_{i} F_{j}\right] u_{i j}=-f .
$$

From Lemma 2.2 we also have

$$
\begin{aligned}
& F_{i} u_{i}=F, \\
& F_{i j} u_{j}=0, \\
& F_{i j k} u_{i}=-F_{j k} .
\end{aligned}
$$

Therefore, making use of (2.7), (2.8) and (2.9), we evaluate

$$
\begin{aligned}
a_{i j} P_{i j}= & p(p-1) F^{2 p-3} F_{l} F_{k} F_{i j} u_{k i} u_{l j} u^{-\beta} \\
& +p(p-1)^{2} F^{2 p-4} F_{l} F_{k} F_{i} F_{j} u_{k i} u_{l j} u^{-\beta} \\
& +p F^{2 p-2} F_{i j} F_{k l} u_{l j} u_{k i} u^{-\beta} \\
& +p(p-1) F^{2 p-3} F_{i} F_{j} F_{k l} u_{l j} u_{k i} u^{-\beta}+p F^{p-1} F_{k} a_{i j} u_{k i j} u^{-\beta} \\
& -2 \beta p F^{2 p-2} F_{i j} F_{k} u_{k i} u^{-\beta-1} u_{j}-2 \beta p(p-1) F^{2 p-3} F_{i} F_{j} F_{k} u_{k i} u^{-\beta-1} u_{j} \\
& +\beta(\beta+1) F^{2 p-1} F_{i j} u^{-\beta-2} u_{i} u_{j} \\
& +\beta(\beta+1)(p-1) F^{2 p-2} F_{i} F_{j} u^{-\beta-2} u_{i} u_{j} \\
& +\beta F^{p} u^{-\beta-1} f .
\end{aligned}
$$


On the other hand, from (2.6) one may easily derive the following identities

$$
\begin{aligned}
& F_{k} u_{k i}=\frac{P_{i}}{p F^{p-1}} u^{\beta}+\beta F \frac{u_{i}}{p u}=\beta F \frac{u_{i}}{p u}+\text { terms containing } P_{k}, \\
& F_{i} F_{k} u_{k i}=\frac{P_{i} F_{i}}{p F^{p-1}} u^{\beta}+\beta \frac{F^{2}}{p u}=\beta \frac{F^{2}}{p u}+\text { terms containing } P_{k} .
\end{aligned}
$$

Moreover, making use of (2.12) in (2.8), we obtain

$$
F^{p-1} F_{i j} u_{i j}=-f-\beta \frac{p-1}{p} \frac{F^{p}}{u}+\text { terms containing } P_{k} .
$$

Differentiating (2.8), we also have

$$
\begin{aligned}
-f^{\prime} u_{k}= & 2(p-1) F^{p-2} F_{i l} F_{j} u_{l k} u_{i j} \\
& +(p-1) F^{p-2} F_{l} F_{i j} u_{l k} u_{i j}+F^{p-1} F_{i j l} u_{l k} u_{i j} \\
& +(p-1)(p-2) F^{p-3} F_{i} F_{l} F_{j} u_{l k} u_{i j}+a_{i j} u_{i j k}
\end{aligned}
$$

Inserting now $a_{i j} u_{i j k}$ from (2.14) into (2.10) and making use of (2.11)-(2.12) we obtain

$$
\begin{aligned}
a_{i j} P_{i j}= & p(p-1) F^{2 p-3} F_{l} F_{k} F_{i j} u_{k i} u_{l j} u^{-\beta} \\
& +p(p-1)^{2} F^{2 p-4}\left(\frac{\beta F^{2}}{p u}+\text { terms containing } P_{k}\right)^{2} u^{-\beta} \\
& +p F^{2 p-2} F_{i j} F_{k l} u_{l j} u_{k i} u^{-\beta} \\
& +p(p-1) F^{2 p-3} F_{i} F_{j} F_{k l} u_{l j} u_{k i} u^{-\beta}-p F^{p} f^{\prime} u^{-\beta} \\
& -p(p-1)(p-2) F^{2 p-4}\left(\frac{\beta F^{2}}{p u}+\text { terms containing } P_{k}\right)^{2} u^{-\beta} \\
& -p(p-1) F^{2 p-3} F_{l} F_{k} F_{i j} u_{l k} u_{i j} u^{-\beta} \\
& -2 p(p-1) F^{2 p-3} F_{j} F_{k} F_{i l} u_{l k} u_{i j} u^{-\beta} \\
& -p F^{2 p-2}\left(\beta u_{l} \frac{F}{p u}+\text { terms containing } P_{k}\right) F_{i j l} u_{i j} u^{-\beta} \\
& -2 \beta p(p-1) F^{2 p-2}\left(\beta \frac{F^{2}}{p u}+\text { terms containing } P_{k}\right) u^{-\beta-1} \\
& +\beta(\beta+1)(p-1) F^{2 p} u^{-\beta-2}+\beta F^{p} f u^{-\beta-1} .
\end{aligned}
$$


Moreover, using (2.9) and (2.13) in (2.15), after some simplifications we get

$$
\begin{aligned}
a_{i j} P_{i j}= & p F^{2 p-2} F_{i j} F_{k l} u_{l j} u_{k i} u^{-\beta}+\beta^{2} \frac{p-1}{p} F^{2 p} u^{-\beta-2} \\
- & p F^{p} f^{\prime} u^{-\beta}-(p-1) \beta F^{p}\left[-f-\beta \frac{p-1}{p} \frac{F^{p}}{u}\right] u^{-\beta-1} \\
& +\beta F^{p}\left[-f-\beta \frac{p-1}{p} \frac{F^{p}}{u}\right] u^{-\beta-1}-2 \beta^{2}(p-1) F^{2 p} u^{-\beta-2} \\
& +\beta(\beta+1)(p-1) F^{2 p} u^{-\beta-2} \\
+ & \beta F^{p} f u^{-\beta-1}+\text { terms containing } P_{k} \\
= & p F^{2 p-2} F_{i j} F_{k l} u_{l j} u_{k i} u^{-\beta}+\beta(p-1) F^{2 p} u^{-\beta-2}\left[\beta \frac{p-1}{p}-\beta+1\right] \\
+ & F^{p} u^{-\beta}\left[-p f^{\prime}+\beta(p-1) \frac{f}{u}\right]+\text { terms containing } P_{k} .
\end{aligned}
$$

Next, making use of $(2.8),(2.11)$ and $(2.12)$, we evaluate separately the term $F^{2 p-2} F_{i j} F_{k l} u_{l j} u_{k i}$, as follows:

$$
\begin{aligned}
& F^{2 p-2} F_{i j} F_{k l} u_{l j} u_{k i}=\left[a_{i j}-(p-1) F^{p-2} F_{i} F_{j}\right] \\
& \times\left[a_{k l}-(p-1) F^{p-2} F_{k} F_{l}\right] u_{l j} u_{k i} \\
& =a_{i j} a_{k l} u_{l j} u_{k i}+(p-1)^{2} F^{2 p-4}\left(\beta \frac{F^{2}}{p u}+\text { terms containing } P_{k}\right)^{2} \\
& \quad-2(p-1) F^{2 p-4}\left[F F_{k l}+(p-1) F_{k} F_{l}\right] \\
& \times\left(\beta \frac{F u_{l}}{p u}+\text { terms containing } P_{k}\right)\left(\beta \frac{F u_{k}}{p u}+\text { terms containing } P_{k}\right) \\
& =a_{i j} a_{k l} u_{l j} u_{k i}-\beta^{2}\left(\frac{p-1}{p}\right)^{2} \frac{F^{2 p}}{u^{2}}+\text { terms containing } P_{k} .
\end{aligned}
$$

Inserting now (2.17) into (2.16), we obtain

$$
\begin{aligned}
a_{i j} P_{i j} & =p u^{-\beta} a_{i j} a_{k l} u_{l j} u_{k i}+\beta(1-\beta)(p-1) F^{2 p} u^{-\beta-2} \\
& +F^{p} u^{-\beta}\left[-p f^{\prime}+\beta(p-1) \frac{f}{u}\right]+\text { terms containing } P_{k} .
\end{aligned}
$$

Next, to evaluate the term $a_{i j} a_{k l} u_{l j} u_{k i}$ in (2.18), we make use of Lemma 2.3 
and identity (2.12). We thus obtain

$$
\begin{aligned}
a_{i j} a_{k l} u_{l j} u_{k i} & \geq \frac{f^{2}}{N}+\frac{N}{N-1}\left[\frac{f}{N}+\beta \frac{p-1}{p} \frac{F^{p}}{u}+\text { terms containing } P_{k}\right]^{2} \\
& =\frac{f^{2}}{N-1}+2 \beta \frac{1}{N-1} \frac{p-1}{p} F^{p} \frac{f}{u} \\
& +\beta^{2} \frac{N}{N-1}\left(\frac{p-1}{p}\right)^{2} \frac{F^{2 p}}{u^{2}}+\text { terms containing } P_{k}
\end{aligned}
$$

Therefore, inserting (2.19) into (2.18), we obtain

$$
\begin{aligned}
a_{i j} P_{i j} & \geq \frac{p}{N-1} \frac{f^{2}}{u^{\beta}}+\frac{F^{p}}{u^{\beta}}\left[\beta(p-1) \frac{N+1}{N-1} \frac{f}{u}-p f^{\prime}\right] \\
& +\beta^{2}(p-1) \frac{F^{2 p}}{u^{\beta+2}}\left[1-\beta \frac{N-p}{p(N-1)}\right]+\text { terms containing } P_{k} .
\end{aligned}
$$

Finally, we analyze separately the case when $1<p<N$, respectively the case when $p \geq N$.

I. The case $1<p<N$ :

In this case $\beta=p \frac{N-1}{N-p}$, so that we have

$$
1-\beta \frac{N-p}{p(N-1)}=0 .
$$

On the other hand, condition (1.4) implies in this case

$$
\beta(p-1) \frac{N+1}{N-1} \frac{f}{u}-p f^{\prime} \geq 0 .
$$

Therefore, making use of (2.21) and (2.22) in (2.20), we are lead to the following elliptic second order differential inequality

$$
a_{i j} P_{i j}+\text { terms containing } P_{k} \geq 0 \text { in } \Omega \text {. }
$$

The conclusion of Theorem 2.1 follows now as a direct consequence of Hopf's first maximum principle (see R. Sperb [15]).

II. The case $p \geq N$ :

In this case $\beta=L p(N-1) /(p-1)(N+1) \geq 0$, so that we have

$$
1-\beta \frac{N-p}{p(N-1)} \geq 1 \text {. }
$$


Also, condition (1.4) implies (2.22).

Using (2.24) and (2.22) in (2.20), we see again that $P$ satisfies an elliptic differential inequality of type (2.23), therefore the proof of Theorem 2.1 is thus achieved.

\section{The proof of Theorem 1.1}

For the proof we adapt an idea employed by L.A. Caffarelli, N. Garofalo and F. Segala [1] to obtain a different Liouville type theorem (see, also, the recent works of A. Farina and E. Valdinoci [8], respectively M. Cozzi, A. Farina and E. Valdinoci [2]).

We first note that $u \in C_{l o c}^{1, \alpha}\left(\mathbb{R}^{N}\right), \alpha \in(0,1)$ and $u \in C^{3}(\{\nabla u \neq 0\}$ ) (for clear proofs of these statements, see Proposition 3.1, Proposition 3.2 and Appendix A in M. Cozzi-A. Farina-E. Valdinoci [2]). Then we introduce the following set

$$
\mathcal{S}_{u}:=\left\{v \text { satisfies }(1.3) ; \inf _{\mathbb{R}^{N}} u(\mathbf{x}) \leq v(\mathbf{x}) \leq \sup _{\mathbb{R}^{N}} u(\mathbf{x}) \forall \mathbf{x} \in \mathbb{R}^{N}\right\},
$$

and remark thus that it is compact in the topology of $C_{l o c}^{1, \alpha}\left(\mathbb{R}^{N}\right)$. Now, let us define

$$
P_{0}:=\sup _{\substack{v \in \mathcal{S}_{u} \\ \mathbf{x} \in \mathbb{R}^{N}}} P(v ; \mathbf{x})<\infty .
$$

We claim that $P_{0} \equiv 0$. From this, Theorem 1.1 follows immediately. To this end, we argue by contradiction and assume contrariwise that

$$
P_{0}>0 \text {. }
$$

By (3.2), there exist two sequences, $\left(v_{k}\right)_{k \in \mathbb{N}} \subset \mathcal{S}_{u}$ and $\left(\mathbf{x}_{k}\right)_{k \in \mathbb{N}} \subset \mathbb{R}^{N}$, such that

$$
\lim _{k \rightarrow \infty} P\left(v_{k}, \mathbf{x}_{k}\right)=P_{0}>0 .
$$

Let us introduce the following functions

$$
w_{k}(\mathbf{x}):=v_{k}\left(\mathbf{x}+\mathbf{x}_{k}\right), k \in \mathbb{N} .
$$

Then, we obviously have

$$
w_{k} \in \mathcal{S}_{u}, P\left(v_{k}, \mathbf{x}_{k}\right)=P\left(w_{k}, \mathbf{0}\right), \lim _{k \rightarrow \infty} P\left(v_{k}, \mathbf{x}_{k}\right)=\lim _{k \rightarrow \infty} P\left(w_{k}, \mathbf{0}\right)=P_{0} .
$$


Moreover, up to a subsequence, we can suppose that there exists $w$ which belongs to $C_{\text {loc }}^{1, \alpha}\left(\mathbb{R}^{N}\right) \bigcap \mathcal{S}_{u}$, such that

$$
\lim _{k \rightarrow \infty} w_{k}=w \text { and } \lim _{k \rightarrow \infty} P\left(w_{k}, \mathbf{0}\right)=P(w, \mathbf{0})=P_{0}>0
$$

In particular, we have that $F(\nabla w(\mathbf{0})) \neq 0$, so that $\nabla w(\mathbf{0}) \neq 0$. By continuity, there exists $\rho>0$ such that

$$
\frac{\inf }{B_{\rho}(\mathbf{0})}|\nabla w(\mathbf{x})|>0
$$

On the other hand, from the definition of $P_{0}$, we know that

$$
P(w ; \mathbf{x}) \leq P_{0}=P(w, \mathbf{0}) \text { for all } \mathbf{x} \in B_{\rho}(\mathbf{0}),
$$

so that $\mathbf{0}$ is a local maximum for $P(w, \cdot)$ in $B_{\rho}(\mathbf{0})$. Theorem 2.1 then implies that $P(w ; \cdot)$ is identically constant in $B_{\rho}(\mathbf{0})$. By the continuity of $P(w ; \cdot)$ and connectedness arguments, we deduce that $P(w ; \cdot)$ is identically constant on the whole of $\mathbb{R}^{N}$, i.e.

$$
P(w ; \mathbf{x}):=\frac{F^{p}(\nabla w(\mathbf{x}))}{w^{\beta}(\mathbf{x})} \equiv P_{0}>0 \text { for all } \mathbf{x} \in \mathbb{R}^{N} .
$$

Now, by the boundedness of $w$ on $\mathbb{R}^{N}$, we must have

$$
\inf _{\mathbb{R}^{N}}|\nabla w(\mathbf{x})|=0
$$

Let $\left(\mathbf{y}_{k}\right)_{k \in \mathbb{N}} \subset \mathbb{R}^{N}$ be a sequence such that $\left|\nabla w\left(\mathbf{y}_{k}\right)\right| \rightarrow 0$ as $k \rightarrow \infty$. By (3.10), we have

$$
\frac{F^{p}\left(\nabla w\left(\mathbf{y}_{k}\right)\right)}{w^{\beta}\left(\mathbf{y}_{k}\right)}=P_{0}>0
$$

Letting $k \rightarrow \infty$ in (3.12) and taking into account that $\inf _{\mathbb{R}^{N}} w(\mathbf{x})>0$, we get

$$
P_{0}=0
$$

which contradicts our assumption. Consequently, $P_{0} \equiv 0$ on $\mathbb{R}^{N}$, which implies that

$$
\nabla u(\mathbf{x}) \equiv \mathbf{0} \text { for all } \mathbf{x} \in \mathbb{R}^{N} .
$$

The proof of Theorem 1.1 is thus achieved. 


\section{Some final remarks. An alternative approach}

Writing $Q(u)$ as $\operatorname{div}(A(\nabla u))$, one may easily check that $Q$ is a $S$-p-C operator (see [3], p. 971) and has the weak Harnack property (see Lemma A.2 in [5]). Moreover, since we have assumed that the solution is bounded away from 0 , that is $0<c<u(\mathbf{x})<d<+\infty$, for some constants $c$ and $d$, then the only thing that has a role is the expression of $f$ on the interval $[c, d]$. Outside this interval, one may modify the function $f$ so that it satisfies our condition (1.4). Therefore, results from some recent results of L. D'Ambrosio and E. Mitidieri (see [3], [4] and [5]) also apply to our problem. More precisely, let us rewrite condition (1.4) of $f$ as

$$
f^{\prime}(t) \leq l \frac{f(t)}{t} \text { for any } t \in \mathbb{R},
$$

where $l:=(p-1)(N+1) /(N-p)$, when $1<p<N$, and $l$ is a nonnegative real constant, when $p \geq N$. Let us also consider the following three sets

$$
P:=\{t: f(t)>0\}, \quad Z:=\{t: f(t)=0\}, \quad N:=\{t: f(t)<0\} .
$$

Then, each of these sets, if not empty, is an interval and

$$
P=(0, \beta), \quad Z=[\beta, \gamma], \quad N=(\gamma,+\infty),
$$

with $0<\beta \leq \gamma \leq+\infty$. Indeed, let us assume that $f\left(t_{0}\right)>0$ and let $I$ be the maximal interval such that $t_{0} \in I$ and $f>0$ on $I$. We will show that inf $I=0$; in such a case, $P$ would be an interval of the form $(0, \beta)$. To this end, let us assume that $\alpha:=\inf I>0$ and let us consider $s<\tau$ in $I$. Integrating (4.1) from $s$ to $\tau$ we get $f(\tau) \leq f(s) \tau^{l} / s^{l}$. Letting $s \rightarrow \alpha$, since $f(s) \rightarrow 0$ ( $f$ is continuous and $I$ is maximal), we have $f(\tau) \leq 0$ for all $\tau \in I$, contradicting thus the fact that $f\left(t_{0}\right)>0$. Analogously, one may prove that $N=(\gamma,+\infty)$, if $N \neq \emptyset$.

Therefore, if $u$ is a weak solution of (1.3), from L. D'Ambrosio and E. Mitidieri's works [3], [4], [5] we get that $u \leq \gamma$. On the other hand, since inf $u>0$, from [3] we have $u \geq \beta$ so that weak Harnack inequality implies that $u$ is constant a.e. in $\mathbb{R}^{N}$ and $u \in Z$.

\section{References}

[1] L.A. Caffarelli, N. Garofalo, F. Segala, A gradient bound for entire solutions of quasi-linear equations and its consequences, Comm. Pure Appl. Math., 47 (1994), 1457-1473 . 
[2] M. Cozzi, A. Farina, E. Valdinoci, Gradient bounds and rigidity results for singular, degenerate, anisotropic partial differential equations. arXiv preprint arXiv:1305.2303 (2013).

[3] L. D'Ambrosio, E. Mitidieri, A priori estimates, positivity results, and nonexistence theorems for quasilinear degenerate elliptic inequalities, Adv. Math., 224 (2010), 967-1020.

[4] L. D'Ambrosio, E. Mitidieri, A priori estimates and reduction principles for quasilinear elliptic problems and applications, Adv. Diff. Eqs, 17(9-10) (2012), 935-1000.

[5] L. D'Ambrosio, E. Mitidieri, Liouville theorems for elliptic systems and applications, J. Math. Anal. Appl., 413 (2014), 121-138.

[6] D. Danielli, N. Garofallo, Properties of entire solutions of non-uniformly elliptic equations arising in geometry and in phase transitions. Calc. Var. PDE's, 15 (2002), 451-491.

[7] A. Farina, Liouville-type theorems for elliptic problems, Handbook of differential equations: stationary partial differential equations, textbf4 (2007), 61-116.

[8] A. Farina, E. Valdinoci, Gradient bounds for anisotropic partial differential equations. Calc. Var. PDE's, (2013), DOI: 10.1007/s00526-013-06059 .

[9] V. Ferone, B. Kawohl, Remarks on Finsler-Laplacian, Proc. Amer. Math. Soc., 137(1) (2008), 247-253.

[10] B. Gidas, J. Spruck, Global and local behavior of positive solutions of nonlinear elliptic equations, Comm. Pure Appl. Math., 34 (1981), 525598.

[11] P. Lindqvist, Notes on p-Laplace equation, University of Jyväskylä - Lectures notes, 2006.

[12] L. Modica, A gradient bound and a Liouville theorem for nonlinear Poisson equations, Commun. Pure Appl. Math., 38 (1985), 679-684.

[13] L.A. Peletier, J. Serrin, Gradient bounds and Liouville theorems for quasilinear elliptic equations, Ann. Scuola Norm. Sup. Pisa Cl. Sci., 5(1) (1978), 65-104.

[14] M.H. Protter, H.F. Weinberger, Maximum principles in differential equations, Springer Verlag (1975). 
[15] R. Sperb, Maximum Principles and Their Applications. Academic Press New York (1981).

[16] G. Wang, C. Xia, An Optimal Anisotropic Poincaré Inequality for Convex Domains, Pacific J. Math., 258(2) (2012), 305-326.

Luminiţa BARBU,

Department of Mathematics,

Ovidius University of Constanta,

Bdul Mamaia 124, 900527 Constanta, Romania.

Email: lbarbu@univ-ovidius.ro

Cristian Enache,

"Simion Stoilow" Institute of Mathematics of the Romanian Academy,

010702, Bucharest, Romania.

Email: cenache23@yahoo.com 